Degenshein. Dholuo Interdentals and Domain-initial Strengthening

\title{
DHOLUO INTERDENTALS: FRICATIVES OR AFFRICATES? EVIDENCE FROM DOMAIN-INITIAL STRENGTHENING
}

\author{
Rachael Degenshein \\ Program in Linguistics and Cognitive Science, Dartmouth College, \\ Hanover, NH, USA \\ degenshein@dartmouth.edu
}

\begin{abstract}
The manner of articulation of $[\theta, \varnothing]$ in Kenya Dholuo is described controversially in the literature. This study uses acoustic data collected from three native speakers to test the hypothesis that $[\theta, ð]$ exhibit positional variation, in accordance with domain-initial strengthening. The results support the hypothesis that $[\theta, \varnothing]$ display more stop-like characteristics in strong position than in weak position. All three speakers show longer closure for $[\theta]$ in word-initial than medial position. There is no significant difference in vowel duration before $[\theta, t]$, suggesting $[\theta]$ is not a fricative. The pre-voicing duration of [ð] follows the hierarchy word-medial > word-initial > IP-initial without focus > IP-initial with focus. This result is consistent with the prediction that longer pre-voicing duration is indicative of a weaker closure. Dholuo interdentals are best described as affricates with differences in articulation in prosodically stronger versus weaker positions.
\end{abstract}

\section{INTRODUCTION}

Dholuo interdentals are normally represented by the IPA symbols $[\theta, \varnothing]$, corresponding to interdental fricatives, although they have been described as "alveolar fricatives" (OkothOkombo, 1982), "dental affricates" (Maddieson, 1984), "dental explosives" (Tucker, 1994), and "interdental spirants" (Bole Odaga, 1997). The phonemic inventory of Dholuo also includes the dental plosives [t,d] (Tucker, 1994). These segments are not in complementary distribution, as illustrated by the following minimal and near minimal pairs:

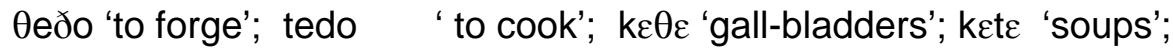

ðans 'human being'; dayo 'to take flesh off the bone'; buðo 'to scorch'; budo 'to soak'

Controversy over the manner of articulation of Dholuo interdentals may be explained by domaininitial strengthening effects. Domain-initial strengthening is the more extreme articulation of a segment at the start of a hierarchical unit of speech (Cho \& Keating, 2001). In general, the higher the position in the prosodic hierarchy the stronger the articulation of the segment. Domain-initial strengthening has been demonstrated in English (Fougeron \& Keating, 1997), French (Fougeron \& Keating, 1996), Taiwanese (Hsu \& Jun, 1998), Estonian (Gordon, 1996), and Korean (Cho \& Keating, 2001) (all as cited in Cho \& Keating, 2001). A more extreme articulation has typically been taken to mean a greater degree of linguopalatal contact (Fougeron \& Keating, 1997). Acoustic effects of strengthening include language-specific lengthening of various elements of a segment. For instance, a study comparing French, English, Taiwanese and Korean found that only in Korean is VOT influenced by prosodic position (Keating et al., 1999). In the same study, only Taiwanese used closure duration to 
Degenshein. Dholuo Interdentals and Domain-initial Strengthening

cumulatively distinguish prosodic positions. Barnes (2002) suggests that domain-initial strengthening may also affect the duration of pre-voicing.

My study attempts to extend these results to the Nilo-Saharan language family. If Dholuo interdentals undergo domain-initial strengthening, then in strong position:

$\mathrm{H} 1$ : In terms of supralaryngeal articulation, closure duration and frication duration will be longer $\mathrm{H} 2$ : In terms of laryngeal articulation, pre-voicing duration will be shorter.

These acoustic characteristics would combine to produce an affricate in stronger prosodic positions and a fricative in weaker prosodic positions. I examine these claims with reference to the strong positions at the beginning of the word and at the beginning of an Intonational Phrase (IP) (following Fougeron \& Keating, 1997). I also attempt to extend the findings to focus effects. In Greek focus words are significantly longer than the same words in neutral sentences (Baltazani \& Jun, 1999). Thus:

H3: Dholuo interdentals occurring domain-initially in focused words may be produced with even greater lengthening than these same segments occurring domain-initially in non-focused words.

\section{EXPERIMENT 1 - POSITION EFFECTS (WORD-INITIAL VS. WORD-MEDIAL)}

\section{Methods}

Experiment 1 examines strengthening effects within the word. I recorded three native male speakers (FO, MO, NB) of Central Nyanzan Dholuo in a soundproof booth at Dartmouth College on a Sony DAT recorder, model TCD-D8 at a sampling rate of $44 \mathrm{kHz}$ using an AKG condenser microphone, model C1000S. I then transferred the files to a computer running Windows 2000, equipped with a digital sound card, and analyzed in Praat 4.0.

The speakers read a list consisting of word-initial and word-medial $[\theta, \chi]$ and $[t, d]$. All words were disyllabic, controlled for tone and location of stress, and matched on vowel height and ATR value. Minimal and near minimal pairs were used whenever possible (see (1)). Each word was embedded in the carrier phrase

$$
\text { ib'iro 'ndiks ___ o o 'ruca (s)he will write }
$$
the day after tomorrow'. (2)

The list was randomized and was read through five times by each speaker yielding 945 distinct utterances. Instances of $[\theta]$ were subsequently analyzed qualitatively and quantitatively. There were too few tokens of word-medial [ð] to warrant positional analysis. The parameters for the quantitative analysis were closure duration and frication duration.

Closure duration was measured as the offset of F2 of the preceding vowel to the start of an identifiable burst, as judged by both the spectrogram and waveform, and was analyzed in a oneway ANOVA with the factors position (2 levels: word-initial, word-medial) and speaker (3 levels: $\mathrm{FO}, \mathrm{MO}, \mathrm{NB})$. Frication duration was measured from the start of the burst to the onset of F1 of the succeeding vowel and analyzed using a one-way ANOVA with the factors position (2 levels: word-initial \& word-medial) and speaker (3 levels: FO, MO, NB). 


\section{Qualitative Analysis}

All speakers reliably produce a pattern characteristic of an affricate, even in the weakest position, word-medial non-pretonic, as shown below. These segments exhibit a period of closure followed by a burst and a period of frication.

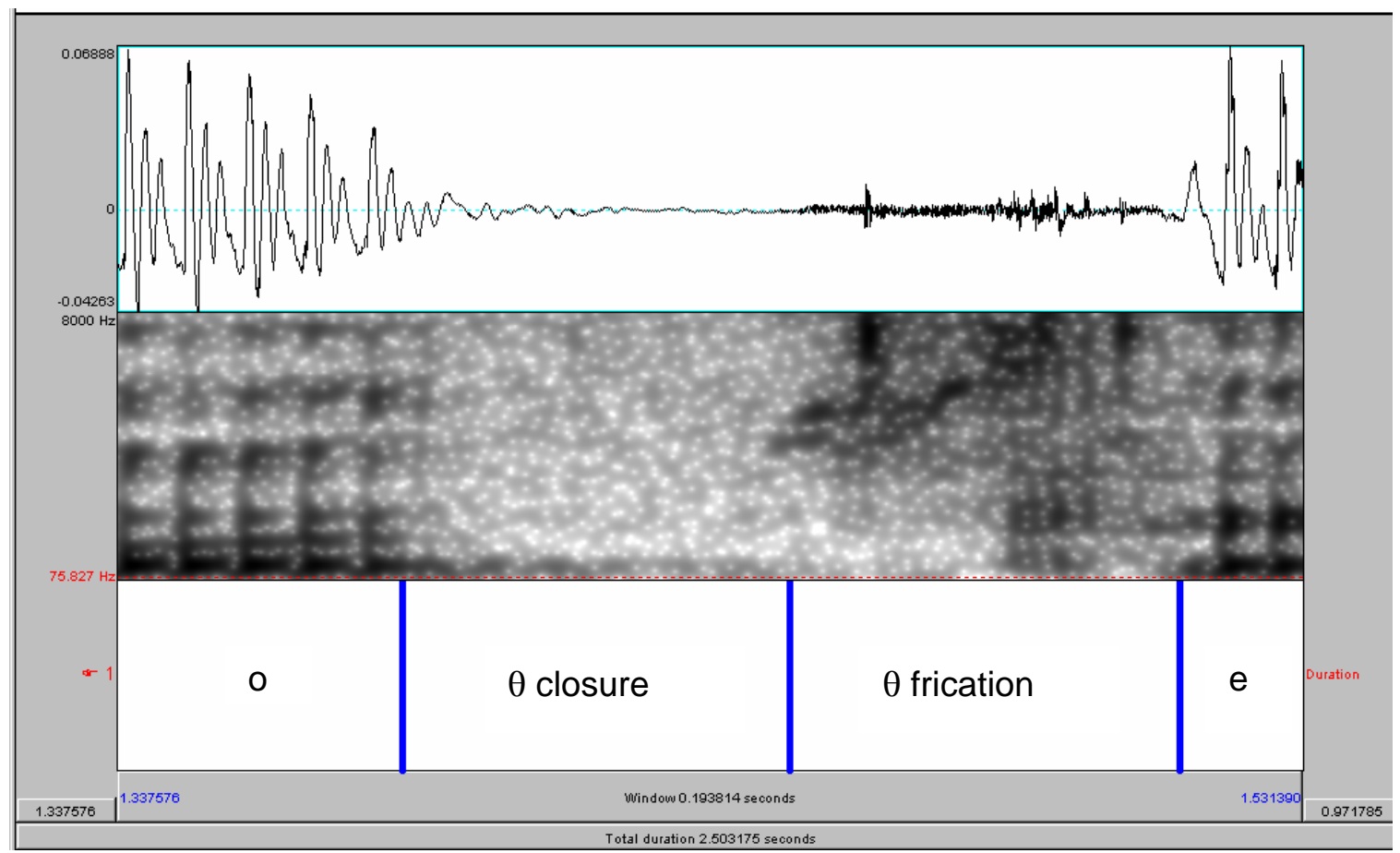

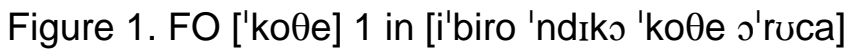

\section{Quantitative Analysis}

Table 1 . Mean closure duration (ms) by speaker

\begin{tabular}{llll}
\hline & word-initial $\theta$ & word-medial $\theta$ & Significance \\
\hline FO & 131.366 & 101.926 & $F(1,88)=45.361, p<.0001$ \\
MO & 161.911 & 127.233 & $F(1,92)=32.462, p<.0001$ \\
NB & 133.198 & 129.703 (n.s.) & $F(1,92)=1.007, p=.318$ \\
\hline
\end{tabular}

There is a significant effect of position on closure duration $(F(1,272)=66.505, p<0.001)$. This effect is significant for $\mathrm{FO}$ and $\mathrm{MO}$ and in the expected direction for NB (see Table1).

There is a significant effect of position $(F(1,272)=9.691, p=.002)$ on frication duration; however, this difference is entirely accounted for by speaker NB. 
Degenshein. Dholuo Interdentals and Domain-initial Strengthening

Table 2. Mean frication duration (ms) by speaker

\begin{tabular}{llll}
\hline & word-initial $\theta$ & word-medial $\theta$ & Significance \\
\hline FO & 60.967 & 61.458 (n.s.) & $F(1,88)=.014, p=.907$ \\
MO & 30.630 & 29.631 (n.s.) & $F(1,92)=.223, p=.638$ \\
NB & 35.359 & 19.550 & $F(1,94)=40.354, p<.0001$ \\
\hline
\end{tabular}

While FO and MO are relatively static across conditions, NB produces nearly double the amount of frication word-initially. NB is the only speaker to significantly vary his frication duration, and is the only speaker not to manipulate closure duration, demonstrating that speakers of Dholuo may use different strategies to realize prosodic strengthening.

\section{EXPERIMENT 2 - VOWEL DURATION}

It is commonly known that vowels are longer before fricatives than before stops. Thus, if $[\theta]$ is a fricative, the vowel preceding it will be longer than the vowel preceding [t]. Only word-medial instances of $[\theta, t]$ were analyzed, to eliminate the confound of the word boundary.

\section{Methods}

The words were recorded in accordance with the methods presented in Exp. 1. Overall, 90 word-medial $[\theta]$ tokens and 75 word-medial $[t]$ tokens were analyzed. I used minimal pairs or near minimal pairs controlling for vowel height, ensuring that a variety of vowel heights were represented in the aggregate. Vowel duration was measured from the onset of F1 to the offset of F2. The results were analyzed in a one-way ANOVA with the factors manner (2 levels: stop, affricate) and speaker (3 levels: FO, MO, and NB). The dependent variable was vowel duration.

\section{Results}

There is no significant effect of manner on the duration of the preceding vowel $(F(1,169)=.003$, $p=.955)$, supporting the hypothesis that $[\theta]$ is not a fricative.

\section{EXPERIMENT 3 - IP AND FOCUS EFFECTS}

\section{Methods}

To test the hypothesis that stronger position corresponds to a stronger closure and hence shorter pre-voicing duration, I examined [ð,d] in four positions:

1) Intonational phrase initial with contrastive focus $(I P+F)$ as in the frame sentence 'ok 'mans, I'ndiks 'kama. 'Not 'mano', should be written this way. (3)

2) Intonational phrase initial without contrastive focus (IP-F) as in the frame sentence 'ok 'kama, I'ndiks 'kama. 'Not this way, should be written this way. (4)

3) Word initial within an IP (identical to Exp. 1 word-initial, denoted W; see (2))

4) Syllable initial in a mid-phrase word (identical to Exp. 1 word-medial, denoted S, see (2). 
Note that there are no instances of [d] in S position due to our control criteria. I hypothesized that pre-voicing will follow the hierarchy $S>W>I P-F>I P+F$, and that [ $\delta$ ] pre-voicing duration will approach [d] pre-voicing duration as the strength of position increases.

The speakers and equipment were identical to those described for Exp. 1. For this experiment, the speakers read a list of words with [ð, d] in word-initial position. All of these words had been previously recorded in Exp. 1. Each of the words was placed in both frame sentence (3) and (4). The sentences were then randomized and read by each speaker five times. The results of Exp. 1 were also factored in, including word-medial tokens for [ð].

Pre-voicing was measured from the onset of the voicing bar during the closure portion to the start of the burst and the results were analyzed using a one-way ANOVA with the factors prosodic position (4 levels: IP+F, IP-F, W, S) and speaker (3 levels: FO, MO, NB) and duration of pre-voicing as the dependent variable.

\section{Results}

There is a significant effect of position on voicing duration for $[ð](F(3,425)=154.863, p<.0001)$. The observed order of pre-voicing, $\mathrm{S}>\mathrm{W}>\mathrm{IP}-\mathrm{F}>\mathrm{IP}+\mathrm{F}$, exactly matches predictions.

Table 4. Mean Pre-voicing duration (ms) of [ð,d] by prosodic position

\begin{tabular}{|l|l|l|l|l|}
\hline & S & W & IP-F & IP+F \\
\hline ə & 117.237 & 111.901 & 75.700 & 70.508 \\
\hline$d$ & N/A & 100.036 & 74.482 & 67.648 \\
\hline
\end{tabular}

Post-hoc tests show that the pre-voicing duration of [ð] is significantly different in all positions, except between $\mathrm{W}$ and $\mathrm{S}(\mathrm{s}=3.32493, \mathrm{p}=.112)$ Thus pre-voicing is a correlate of both positional and focus-based strengthening.

For [d], position has a significant effect on pre-voicing duration $(F(2,477)=172.176, p<.0001)$ and the observed order of pre-voicing $\mathrm{W}>\mathrm{IP}-\mathrm{F}>\mathrm{IP}+\mathrm{F}$ is also in line with predictions. Post-hoc tests reveal that each position differs significantly from all other positions $(s=1.8, p<.0001)$.

The hypothesis that pre-voicing of [ð] will approach that of [d] as strength of position increases is supported by the significant interaction between manner and position in a one-way ANOVA $(F(2,916)=5.520, p=.004)$. The pre-voicing duration of $[0, d]$ do converge as strength of position increases, with a significant difference based on manner in $W$ position $(F(1,291)=25.884$, $\mathrm{p}<.0001)$, and no significant difference between manners in either of the IP-initial positions $(I P+F: F(1,290)=.203, p=.653 ;$ IP-F: $F(1,291)=1.194, p=.275)$.

\section{DISCUSSION}

The results of the present study support the hypothesis that domain-initial strengthening does play a role in the realization of Dholuo interdentals. The longer closure duration of $[\theta]$ in wordinitial than word-medial position supports the notion that closure duration is a correlate of 
strengthening for Dholuo. Additionally, speaker NB's manipulation of frication duration as a marker of prosodic strength underscores the existence of multiple different strategies for recognizing positional variation. The lack of a significant difference in the duration of vowels preceding $[\theta, t]$ argues against the description of $[\theta]$ as a fricative. Finally, evidence at the IP levels indicates that both $[ð, d]$ exhibit progressively shorter pre-voicing duration as strength of position increases. Additionally, the pre-voicing duration of [ð] approaches [d] IP-initially, demonstrating that [ð] exhibits more stop-like characteristics in strong position. This evidence from several different acoustic avenues points to the characterization of Dholuo interdentals as affricates, particularly given their qualitative analysis, and suggests that failure to control for prosodic position may have contributed to the controversial descriptions of these segments.

\section{REFERENCES}

Baltazani, M. \& Jun, S. (1999) Focus and topic intonation in Greek, Proceedings of the Fourteenth ICPhS 2, 1305-1308.

Barnes, J. (2002) Positional neutralization: A phonologization approach to typological patterns: University of California at Berkeley.

Bole Odaga, A. (1997) English-Dholuo Dictionary. Kisumu, Kenya: Lake Publishers \& Enterprises, Ltd.

Cho, T. \& Jun, S. (2000) Domain-initial strengthening as enhancement of laryngeal features: Aerodynamic evidence from Korean, UCLA Working Papers in Phonetics 99 57-69.

Cho, T. \& Keating, P. (2001) Articulatory and acoustic studies on domain-initial strengthening in Korean, Journal of Phonetics 29, 155-190

Fougeron, C. \& Keating, P. (1997) Articulatory strengthening at edges of prosodic domains, Journal of the Acoustical Society of America 101, 3728-3740

Keating, P., Cho, T., Fougeron, C., Hsu, C. (1999) Domain-initial articulatory strengthening in four languages, UCLA Working Papers in Phonetics 97, 139-151.

Maddieson, I. (1984) Patterns of Sounds, New York: Cambridge University Press.

Okoth-Okombo, D. (1982) Dholuo Morphophonemics in a Generative Framework, Berlin: Dietrich Reimer Verlag.

Tucker, A.N. (1994). A Grammar of Kenya Luo (Dholuo) Köln: Rüdiger Köppe Verlag. 